\title{
Origen de los recursos y ventajas competitivas de las organizaciones: reflexiones teóricas
}

\author{
Zapata Rotundo, Gerardo J.* \\ Hernández, Aymara**
}

\section{Resumen}

En el marco de los estudios sobre organización, existen diferentes enfoques que explican el origen de sus recursos y ventajas competitivas. En el presente artículo de carácter teórico, se abordan dos: recursos y capacidades y dependencia de recursos. Así, se lleva a cabo una revisión bibliográfica con el propósito de plantear un modelo teórico integrado combina ambos enfoques y enfatiza su complementariedad y una serie de proposiciones. Estas últimas vinculan el proceso de toma de decisiones para adquirir los recursos necesarios y los factores asociados con la visión directiva y la racionalidad limitada del individuo. Como conclusión más relevante se plantea que la toma de decisiones sobre la adquisición de recursos para la organización no solo depende de procesos racionales orientados por principios administrativos y limitados por la capacidad de aprendizaje y análisis del individuo, sino que también están guiados por la visión del gerente, la cual se expresa a través de su acción voluntaria, subjetiva y discrecional en cuanto a la manera como debe ser organizada y dirigida la empresa.

Palabras clave: Teoría de la organización, teoría de recursos y capacidades, teoría de la dependencia de recursos, ventajas competitivas.

\section{Recibido: 06-12-12 Aceptado: 15-05-14}

* Licenciado en Contaduría Pública. Especialista en Contaduría, Mención Auditoría. Doctor en Organización y Dirección de Empresas, Universitat de València, España. Profesor Titular, Decanato de Administración y Contaduría, Universidad Centroccidental "Lisandro Alvarado", Barquisimeto, Venezuela. E- mail: zapager@yahoo.com.

** Ingeniero en Informática. Magister en Ingeniería Industrial (UNEXPO). Doctora en Estudios del Desarrollo (CENDES-UCV). Profesora Titular del Decanato de Administración y Contaduría de la Universidad Centroccidental “Lisandro Alvarado”, Venezuela. E-mail: aymarah@yahoo. com. 


\title{
The Origin of Resources and Competitive Advantages of Organizations: Theoretical Reflections
}

\begin{abstract}
Within the framework of organizational theory, there are different theoretical approaches that explain the origin of their resources and competitive advantages. Two of these theoretical reflections are addressed in this paper: the theory of resources and capacities and the resource dependence theory. A review of literature was carried out in order to present an integrated theoretical model that combines both approaches and emphasizes their complementarity and a series of propositions. These propositions link the decision-making process to acquiring the required resources and the factors associated with directive vision and the individual's limited rationality. The most relevant conclusion proposes that decision making about resource acquisition for the organization depends on two principal factors: a) rational processes guided by administrative principles and limited by the individual's capacity for learning and analysis, and b) the manager's vision, expressed through his voluntary, subjective and discretionary actions about how the company should be organized and led.
\end{abstract}

Keywords: Organization theory, theory of resources and capabilities, resource dependence theory, competitive advantages.

\section{Introducción}

La teoría de la organización se ha preocupado de manera especial por la línea de investigación que aborda el estudio sobre el origen de los recursos y capacidades de la organización y, como consecuencia de ello, sus ventajas competitivas. Por una parte, se encuentra la corriente paradigmática ubicada dentro de la teoría neoclásica que ha formulado sus postulados alrededor de la relevancia del entorno en relación al destino y supervivencia de la organización, con lo cual se convierte en su principal condicionante. Por la otra, el enfoque teórico que concede a los recursos y capacidades internas un papel de vital importancia a la hora de definir sus ventajas competitivas. En primer orden, la teoría de dependencia de recursos (TDR) y, en segundo orden la de recursos y capacidades (TRC). Enfoques teóricos con posturas diferentes, pero que en esta investigación se trata de encontrar puntos de complementariedad que proporcionen nuevas ideas en el intento de conocer con mayor profundidad y sentido de integración los procesos de desarrollo organizacional.

La teoría de dependencia de recursos, sostiene que la organización no tiene la capacidad, ni es autosuficiente para producir y controlar todos los recursos y servicios que necesita para su crecimiento, mantenimiento o supervivencia; en consecuencia, debe recurrir a su entorno para lograr tales fines. Se crea con esto una dependencia externa limitada por la disponibilidad y la facilidad de acceso a esos recursos. Por esta razón, tiene que adaptar sus estrategias a las características del entorno donde se desenvuelve, 
cuestión que pasa por implementar políticas y estrategias vinculadas con las relaciones de interdependencia (Pfeffer y Salancik, 1978; Ulrich y Barney, 1984; Wei, 2006; Nienhüser, 2008). Dicha teoría hace un énfasis especial al enlace entre los recursos internos, las estrategias y el desempeño de la organización con el entorno donde se desenvuelve. Planteamiento este, enmarcado dentro pensamiento neoclásico de la teoría de la organización, en el entendido que observa al entorno como "dado"; por consiguiente, y bajo esa visión, el proceso de adaptación se convierte casi en el único camino viable para que la empresa pueda sobreponerse a la competencia y, en general, a sus demandas.

La teoría de recursos y capacidades sugiere que los recursos que posee, controla y puede generar la empresa contribuyen de manera sustancial al logro de mayores niveles de desempeño, convirtiéndose, por tanto, en la fuente fundamental de sus ventajas competitivas. Por ello, reivindica el papel que juegan sus características internas como la base esencial para el establecimiento de sus políticas y estrategias (Dierickx y Cool, 1989; Grant, 1991; Barney, 1991, 2001; Song et al., 2005; Yang, 2008; Gupta et al., 2011; Coleman et al., 2013). Esta teoría tiene una visión menos estática y más emprendedora de los elementos internos de la organización con respecto a las premisas de la TDR. De ahí, surgen a primera vista diferencias importantes a la hora de explicar el origen de sus recursos, $y$, por ende, su comportamiento y resultados.

De este modo, este artículo de carácter teórico tiene como objetivo análizar y reflexionar sobre las teorías de recursos y capacidades y de dependencia de recursos. Esto con el propósito de: primero, plantear un modelo teórico integrado del origen de los de recursos y capacidades de la organización y, segundo, ofrecer una serie de proposiciones que vinculan la toma de decisiones relacionadas con la adquisición de recursos y capacidades con los factores asociados a la visión directiva y a la racionalidad limitada del individuo. El interés primordial es resaltar el hecho de que ambas teorías pueden resultar complementarias y no excluyentes como tradicionalmente han sido tratadas y aplicadas cuando se evalúa el origen y desarrollo de los recursos y capacidades de la organización. En definitiva, y por extensión, ambas teorías han contribuido a explicar el comportamiento, la estructura, los procesos, los resultados y, en general, los cambios que ocurren en la organización, constituyéndose en uno de los principales soportes para el desarrollo de la teoría organizacional.

Desde el punto de vista metodológico, esta investigación tiene carácter teórico-descriptivo basada en una revisión bibliográfica en función al objetivo propuesto. El desarrollo del artículo se sustenta en el análisis, ordenamiento y síntesis de los principios y postulados de las teorías que explican, bajo diferentes enfoques, el origen de los recursos y capacidades de las organizaciones. Como consecuencia de ello, se desarrolla un cuerpo de reflexiones y proposiciones susceptibles de contrastación empírica en investigaciones posteriores de carácter cuantitativo. 


\section{Teoría de recursos y capacidades}

La teoría de recursos y capacidades (TRC), centra su análisis en la importancia de los recursos y capacidades internas de la organización como fuente principal de sus ventajas competitivas, prestándole poca atención a los factores del entorno donde se desenvuelve. Esta teoría ha tenido varias ramificaciones y denominaciones en función a los distintos puntos de vista de los investigadores que la abordan: visión basada en recursos (Wernerfelt, 1984; Oliver, 1997; Barney y Arikan 2001; Barney, 2001; Barney et al., 2001; Priem y Butler, 2001; Hoopes et al., 2003; Zhuang y Lederer, 2006; Bingham y Eisenhardt, 2008; Duh, 2010; Gupta et al., 2011; Coleman et al., 2013), teoría de las ventajas competitivas (Grant, 1991; Ray et al., 2004), teoría de las capacidades dinámicas (Makadok, 2001; Helfat y Peteraf, 2003; Lei, 2003; Hou, 2008), perspectiva de las capacidades de la organización (Langlois y Foss, 1999) y visión basada en el conocimiento (Grant, 1996; Conner y Prahalad, 1996; Nonaka, y Toyama, 2005; Halawi et al., 2005; Ziesemer, 2013; Blome et al., 2014.).

Sin embargo, los autores, en términos generales han manifestado el hecho que las habilidades de la empresa para obtener, generar y controlar recursos escasos, inimitables o pocos sustituibles le proporcionaran mayores posibilidades de desempeños superiores sobre sus competidores, así como de alcanzar una mejor adaptación a las exigencias del entorno, y, por tanto, le permitirán lograr mayores ventajas competitivas sostenibles en el tiempo (Dierick y Cool, 1989; Grant,
1991; Barney, 1991; Oliver, 1997; Tushar y Teng, 2000; Barney, 2001; Esteve y Mañez, 2008; Morris et al., 2010; Gupta et al., 2011; Hsu y Zledonis, 2013).

La TRC proporciona una explicación a la heterogeneidad de la competencia entre las organizaciones. Se parte de la premisa que los participantes difieren en sus recursos y capacidades de manera importante, lo cual determina la presencia de ventajas competitivas (Tushar y Teng, 2000; Helfat y Peteraf, 2003), convirtiéndose en una de las principales teorías para explicar y comprender el origen de los recursos y capacidades de la organización (Barney et al., 2001; Hoopes et al., 2003; Bingham y Eisenhardt, 2008; Morris et al., 2010; Gupta et al., 2011), teniendo contribuciones significativas en el campo de los recursos humanos, la economía, la administración y el marketing (Barney et al., 2001; Helfat y Peteraf, 2003; Ray et al., 2005; Yang, 2008; Morris et al., 2010; Coleman, et al., 2013) y en la internacionalización de la empresa (Escandón y Hurtado, 2013; Gaur et al., 2014). Así, se concluye en algunas investigaciones alrededor de la TRC que la supervivencia de una empresa y su crecimiento dependen en parte de su capacidad para crear nuevos recursos, desarrollarlos y mantenerlos, $y$, además, de cómo los conservan y protegen (Barney, 2001; Ray et al., 2005; Yang, 2008; Gupta et al., 2011).

Los trabajos que iniciaron la formación de los postulados de la teoría de recursos y capacidades, se remontan a la década de los años ochenta. Nacen como una respuesta a los argumentos desarrollados hasta ese momento que sostenían la idea de que la supervivencia 
de la organización dependía fundamentalmente de su capacidad de mantener adecuadas relaciones de dependencia con su entorno, relegando a un segundo plano su ámbito interno. Es decir, aquellos enfoques teóricos que conceptualizan el entorno como la fuente principal de recursos para la organización, resaltando que sus exigencias le imponen una serie de condicionantes o factores de contingencia que determinan en buena medida su comportamiento, diseño estructural, procesos de formación e implantación de estrategias, y, en general, en el logro de un adecuado desempeño y de ventajas competitivas (Pfeffer y Salancik, 1978; Donaldson, 2001; Wei, 2006; Nienhüser, 2008). Esto es lo que se denomina el modelo de adaptación, donde se ubica la teoría contingente ${ }^{1}$ y la dependencia de recursos.

Entre los primeros trabajos que iniciaron la formación de los cimientos de la teoría centrada en la explicación de las ventajas competitivas, a partir de la dotación de recursos tangibles e intangibles, se encuentran los de Wernerfelt (1984), Barney (1986), Dierickx y Cool (1989), Prahalad y Hamel (1990), Grant (1991), Barney (1991) y Peteraf (1993). Si bien se le acuña al primer autor el término de visión basada en recursos (RBV, siglas en inglés), se consideran estos estudios una extensión de los trabajos seminales de
Penrose (1959) y Selznick (1957). El primero, definió a la empresa como un conjunto de recursos a la disposición de sus empleados, cuyo uso está determinado por las decisiones, las acciones y la discrecionalidad de los gerentes y directivos, y el segundo quien incorpora el término de "competencia distintiva".

Se plantea la necesidad de que la empresa tiene que observarse de manera muy especial en términos de las posibilidades ofrecidas por sus características internas, y no privilegiar la posición producto-mercado, o enfoque de adaptación, prevaleciente hasta los años ochenta del siglo pasado, como la base esencial de las fuentes de recursos $y$, por consiguiente, el logro de ventajas competitivas. Convirtiendo a la organización, bajo estos planteamientos, en la principal unidad de análisis.

Como referencia conceptual, se toma la definición de recursos como a todos aquellos activos, procesos, características organizacionales y conocimientos controlados por la empresa, que le permiten, a su vez, alcanzar su éxito con la mayor eficiencia posible (Barney, 1991; Zhuang y Lederer, 2006; Yang, 2008; Morris et al., 2010). En otros trabajos, Barney (2001) y Barney y Arikan (2001) incorporan el concepto de activo tangible e intangible; y consistente con esas ideas Bingham y Eisenhardt (2008),

1 En las bases del planteamiento contingente-modelo de adaptación -, está el supuesto de la existencia de una serie de factores internos y externos a la organización que actúan como variables causales o explicativas de su diseño. Las organizaciones que alcancen un mejor ajuste y adaptación con esos factores de contingencia, serán las más eficientes y tendrán mayor probabilidad de supervivencia (Donalson, 2001; Aragón-Correa y Sharma, 2003; Volberda, et al., 2012; Akingbola, 2013). 
además de considerar los activos tangibles e intangibles, resaltan la importancia de los procesos organizacionales como recursos de la empresa ${ }^{2}$.

La literatura de TRC ha definido de modo general el término de capacidades, como el conjunto de habilidades profesionales y el conocimiento acumulado (tácito y explícito) ejercido a través de los procesos de la organización que permiten coordinar las actividades y aprovechar el uso más adecuado de los activos con el propósito de alcanzar los objetivos trazados (Day, 1994; Song, et al., 2005; Yang, 2008; Duh, 2010; Molloy, et al., 2011). Con anterioridad, Grant (1991) en su trabajo seminal, argumentaba que la capacidad consiste en obtener ciertos resultados a través de la coordinación y la aplicación de las habilidades de los miembros de la organización sobre un grupo de recursos ${ }^{3}$. En ese mismo orden de ideas, Collis (1994) ha expresado que la utilización en conjunto de los activos tangibles e intangibles en combinación con el conocimiento tecnológico y organizativo, permite desarrollar capacidades operativas y gerenciales altamente innovadoras

Se desprende entonces la idea de que los recursos tangibles están constituidos por el capital físico y financiero que posee y controla la organización, y que pueden ser reflejados con cierta facilidad en sus estados financieros. En el intangible, por ejemplo, se incluye el capital humano y el organizacional. El primero de ellos se refiere a la experiencia, los conocimientos, la tecnología, know-how, y la formación de los recursos humanos. Y el capital organizacional, lo conforma la cultura, la lealtad de los clientes, la reputación, la estructura organizativa y los procesos organizativos. Cabe señalar que los intangibles tienen un origen particular e idiosincrásico asociados e inherentes a la dinámica de la propia empresa, con lo cual se convierten en el principal factor de diferenciación y heterogeneidad entre las organizaciones (Hoopes et al., 2003; Bingham y Eisenhardt, 2008), y sobre todo cuando se adquiere la capacidad de crear, aprender, integrar y transferir el conocimiento. Convirtiéndose la gestión del conocimiento en una de las ramificaciones de la TRC, y una de las principales funciones de las organizaciones que pretenden desarrollar capacidades diferenciadoras (Conner y Prahalad, 1996; Grant, 1996; Nonaka, 1994; Nonaka y Takeuchi, 1995; Lei, 2003; Nonaka y Toyama, 2005; Abenson y Taku, 2006; Blome et al., 2014).

Por sus características, los activos intangibles son en primera instancia los recursos que puede desarrollar la organización, además que resultan los más difí-

2 Procesos organizacionales necesarios, por ejemplo, para desarrollar nuevos productos y servicios o formas de entrada en nuevos mercados que crean valor estratégico y ventajas competitivas (Bingham y Eisenhardt, 2008).

3 Grant (1991:122) en su trabajo clásico, manifestaba que "no existe una función predeterminada para el uso de los recursos y capacidades, por lo que un recurso por sí solo no necesariamente genera ventaja competitiva, sino que requiere la combinación y coordinación de equipos de trabajo en la empresa". 
ciles de adquirir, reproducir o imitar sobre todo si se trata de recursos humanos, debido al grado de especialización, habilidades y conocimiento tácito que poseen, a veces difíciles de ser transferidos o copiados ${ }^{4}$. Por estas razones, son uno de los principales generadores de ventajas competitivas sostenibles en el tiempo cuando se aprende a gestionarlos adecuadamente.

En su trabajo seminal, Barney (1991) indica que no todos los recursos son generadores de ventajas competitivas. En ese sentido, este autor señala que deben cumplir con cuatro atributos:

- Valioso. Permiten a la empresa implementar estrategias que mejoren su eficiencia, por ejemplo, optimizar los costos o incrementar los beneficios.

- Raro. Lo poseen, controlan y administran muy pocas organizaciones ${ }^{5}$.

- Inimitable. No reproducible con facilidad, habiendo varios factores para ello: la historia de la empresa, el desarrollo de sus propios recursos a través de procedimientos y técnicas aprendidas, y la interconexión o la interdependencia de los recursos para producirlos, teniendo, en consecuencia, un carácter idiosincrático.

- No equivalente estratégicamente, valioso y no imitable. En efecto, si una empresa tiene recursos estratégicamente equivalentes, puede implemen- tar las mismas estrategias de diferentes maneras con distintos recursos, lo que pone en peligro la sostenibilidad de una ventaja competitiva.

En síntesis, las investigaciones dentro del marco de la TRC han convenido en que los recursos deben ser imperfectamente imitables y poco reproducibles por las empresas rivales, para que proporcionen realmente ventajas competitivas y desempeños superiores. Desprendiéndose de la revisión de la literatura sobre la TRC, las siguientes cinco premisas principales:

- Las empresas que pueden desarrollar recursos escasos, raros, no sustituibles o difíciles de imitar, adquieren ventajas competitivas sobre sus competidores.

- Las organizaciones son básicamente diferentes en función a los recursos que poseen y de las habilidades que desarrollan a partir de ellos.

- Los recursos pueden no ser perfectamente transferibles entre las organizaciones a corto o mediano plazo. En vista de ello, la heterogeneidad de recursos se convierte en duradera.

- Los recursos proporcionan las orientaciones principales para establecer las estrategias organizacionales, siendo las fuentes principales de sus beneficios y ventajas competitivas.

4 Knott (2009) indica que los recursos intangibles los constituyen, además de los recursos humanos, la información y la reputación de la empresa, otra clase recursos intangibles como las capacidades y las competencias.

5 Al respecto, Hamel y Prahalad (1995) señalan que un recurso con mucha disponibilidad en el mercado puede ser un requisito importante para competir, pero no se convierte necesariamente en una fuente de ventajas competitivas. 
- La heterogeneidad y ventajas competitivas, son sostenibles en la medida que los competidores no sean capaces de duplicar los beneficios que se derivan de los recursos valiosos, raros inimitables y no sustituibles.

Así, la idea central de la teoría de recursos y capacidades es que la heterogeneidad entre las organizaciones surge de las diferencias que hay en el conjunto de recursos y capacidades que poseen, principalmente con un carácter idiosincrático e inherente a su propia dinámica. Constituyéndose, a su vez, en la base esencial de ventajas competitivas que producen resultados diferentes entre las organizaciones, aún estando ubicadas en un mismo sector económico o industrial y en condiciones contextuales muy semejantes.

\section{Teoría de la dependencia de recursos}

Los trabajos seminales de Aldrich y Pfeffer (1976) y Pfeffer y Salancik (1978), proporcionaron las primeras referencias de la teoría de la dependencia de recursos (TDR). Sostienen estos autores como premisa, que la organización no tiene las habilidades y competencias suficientes para generar todos los recursos y servicios que requiere para mantener sus ventajas competitivas o simplemente para poder sobrevivir. Por ello, se ve en la necesidad, y en algunos casos en la obligación, de establecer relaciones de intercambio con otras organizaciones o factores del entorno (Pfeffer y Salancik, 1978; Ulrich y Barney, 1984; Fink et al., 2006; Nienhüser, 2008; Peterson, 2011). Re- sultando, de acuerdo a estos planteamientos, que inevitablemente están ligadas a las condiciones impuestas por el entorno, diferencia fundamental con el enfoque basado en recursos y capacidades.

La necesidad de adquirir recursos escasos y valiosos del entorno, se traduce en una dependencia externa que conduce a la creación de relaciones de intercambio y acuerdos de cooperación. En ese sentido, Pfeffer y Salancik (1978) señalan que hay tres factores fundamentales para determinar esa dependencia:

- El grado de importancia de los recursos, condicionado por los requerimientos y necesidades de la organización para seguir operando.

- La medida en la cual el grupo de interés tiene albedrio sobre la asignación y uso de los recursos disponibles en el entorno. Lo que significa que la dependencia pueda estar determinada por hechos al azar y libre elección.

- El nivel de control sobre los recursos de los grupos interesados, o la cantidad de alternativas que hay sobre ellos para adquirirlos.

Así, la TDR comparte de alguna manera la visión determinista-contingente en cuanto a que el comportamiento, administración y el diseño de la organización está significativamente condicionado por una serie de mecanismos impersonales y objetivos o factores de contingencia, que actúan como limitantes sobre las acciones de los tomadores de decisiones estratégicas (Zapata y Mirabal, 2011).

Se observa que bajo la visión de la TDR, los factores del entorno son cierta- 
mente críticos para comprender lo que sucede dentro de la organización, constituyéndose en los pronosticadores principales de sus características, de sus procesos internos y sus resultados. Por ello, Pfeffer y Salancik (1978:39) sugieren que "la premisa subyacente de la perspectiva de control externo sobre las organizaciones, es que sus actividades y los productos que elabora, se explican a partir del contexto donde se desenvuelven". Factores a los que debe ajustarse la organización para alcanzar un adecuado desempeño o de simplemente poder sobrevivir, llamadas también perspectivas de: (1) adaptación y (2) selección.

La perspectiva de adaptación, donde se enmarca la teoría de dependencia de recursos y la teoría contingente, da por sentado que las organizaciones tienen que ajustarse al cambio, a las exigencias y a la complejidad del entorno. Por otra parte, la perspectiva de selección, plantea que el entorno escoge a las organizaciones que mejor se acoplen a sus condiciones y criterios, rechazando así a las más débiles ${ }^{6}$. Esta perspectiva de selección, se focaliza más en la capacidad de los factores del entorno que en los propios miembros y recursos de la organización para cambiar o influir en su curso de acción. En consecuencia, el comportamiento de la organización se convierte en una necesaria reacción y adaptación a las limitaciones y obligaciones impuestas por los factores del entorno (Gopalakrishnan y Dugal, 1998; Donaldson, 2001; Child et al., 2003; Han, 2007).

En síntesis, bajo la visión de las perspectivas de adaptación y selección, las características internas de la organización y su capacidad para generar y mantener sus propios recursos son de segundo orden, pasando a un plano inferior la visión y actitud de los gerentes y directivos en cuanto a cómo debe ser organizada y diseñada la empresa

Para ilustrar lo comentado en el párrafo anterior, en relación a la perspectiva de adaptación, se hace referencia, por ejemplo, al modelo clásico y seminal de estrategias competitivas desarrollado por Porter (1982). En el mismo, se observa la preponderancia de la influencia del entorno sobre las decisiones de los gerentes cuando se trata de definir las estrategias organizacionales. Sus planteamientos se basan en la existencia de factores exógenos que determinan de manera importante la rentabilidad de la empresa, y, en consecuencia, ésta debe ajustar sus objetivos, procesos y recursos a las condiciones impuestas por: (a) las amenazas de entrada de nuevos competidores; (b) el poder de negociación y el conocimiento sobre los productos, tanto de los compradores como de los proveedores; (c) las presiones ejercidas en el mercado por el

La perspectiva de selección se corresponde a la teoría de ecología de poblaciones. Entre sus premisas postula que el entorno es quien optimiza y escoge los criterios de aptitud de las organizaciones. Surgiendo de esto, que determinadas formas de organización no prosperan puesto que bajo ciertas circunstancias hay otras que compiten satisfactoriamente por los recursos esenciales y disponibles en el entorno (Hannan y Freeman, 1977,1987; Han, 2007). 
ingreso de productos sustitutivos; y (d) la rivalidad entre los competidores.

En el trabajo seminal de Porter (1982), se nota un enfoque con las características del modelo de adaptación que produce un comportamiento reactivo y adaptativo de la organización, cuestión que es observada también en los trabajos de Aldrich y Pfeffer (1976), Pfeffer y Salancik (1978), Hannan y Freeman (1977, 1987), referidos al modelo de control externo de la organización por parte del entorno.

La idea básica del enfoque de la dependencia de recursos, es el manejo adecuado y oportuno de las relaciones con el contexto externo. Significa que normalmente se obtienen recursos a través del intercambio con otras organizaciones, quienes posiblemente los mantienen bajo su control y dominio. Siendo, por tanto, la palabra clave el uso estratégico de la interdependencia. Dicho proceso puede variar de acuerdo a la disponibilidad de recursos y a las necesidades de cada empresa, con lo cual afecta su capacidad para encontrar los elementos que requiere para un funcionamiento adecuado (Zapata y Mirabal, 2011); potenciándose, por consiguiente, las relaciones de interdependencia. De este modo, es un enfoque teórico con amplio alcance para explicar tanto el comportamiento organizacional como las relaciones interorganizacionales (Rao et al., 2007; Bryant, 2011; Peng y Beamish, 2014).

El planteamiento estratégico en cuanto a la gestión del entorno dentro del marco de la TDR va dirigido no solo a la simple adquisición de recursos, sino que también aborda el estudio del conjunto de acciones necesarias para controlarlo. Medidas que pasan por el establecimien- to de estrategias de coordinación, control y administración de las relaciones con otras organizaciones. En general, los recursos son obtenidos y luego gestionados dentro de la empresa con el propósito de minimizar la dependencia, y a su vez maximizarla con respecto a otras (Pfeffer y Salancik, 1978; Ulrich y Barney, 1984; Nienhüser, 2008).

No obstante, hay que considerar que las relaciones interorganizacionales, además de representar un mecanismo de intercambio de recursos y capacidades, también pueden convertirse en una acción que trae consigo la pérdida de autonomía de algunos de sus participantes. Es decir, las consecuencias naturales en una relación entre un solicitante de recursos críticos y su abastecedor son que el dominio y poder se le concede a aquella entidad que controla las condiciones necesarias para el funcionamiento de las otras (Pfeffer y Salancik, 1978; Rao et al., 2007). Entonces las que requieran de tales recursos, tendrán que ceder algún grado de autonomía, quedando, por tanto, vulnerables a las decisiones de aquellas que los proporcionan y como consecuencia se reproduce y crece la dependencia.

Frente a una situación donde los recursos son suficientes y adicionales, puede haber al menos dos alternativas: (a) evitar formalizar nuevos enlaces en la red con el propósito de reducir la dependencia y así conservar una mayor autonomía; o (b) ceder cierta cantidad de recursos con el fin de aumentar la dependencia de otras organizaciones.

Se observa con evidencia que el modelo de la dependencia de recursos, al contrario del enfoque basado en recur- 
sos, considera que los factores del entorno juegan un papel crítico en la definición de las ventajas competitivas de la organización, sus procesos internos y, por tanto, en su desempeño. De este modo, se afirma la idea que para la organización es de vital importancia conocer su grado de dependencia e incertidumbre con respecto a los elementos y las circunstancias del entorno donde se desenvuelve.

En síntesis, las argumentaciones TDR giran en torno a cuatro ideas básicas:

- El entorno provee los recursos y capacidades críticas.

- Las organizaciones son más sensibles al entorno en la medida que requiere de mayores recursos de él.

- La dependencia del entorno y de los recursos que provee, influyen de forma importante en el comportamiento y la acción de la organización.

- La organización trata de influir sobre el entorno con el propósito que los recursos estén disponibles para ella; es decir, depende de él pero pone en práctica estrategias que le permitan controlarlo o al menos de minimizar su dependencia.

\section{Percepción de la incertidumbre del entorno}

De la tecnología, la innovación, la competencia, los cambios del mercado y, en general, de la complejidad-dinamismo del entorno, emana el concepto de incertidumbre. La misma puede conducir a la organización a un desconocimiento y a elevados niveles de dificultad para llevar a cabo el trabajo necesario para la pro- ducción de los bienes y servicios, que a su vez influyen en las acciones y decisiones de los gerentes y directivos. De este modo, si se conoce el nivel de incertidumbre, con seguridad éstos estarán en mejores condiciones para diseñar y poner en práctica las estrategias que permitan encontrar los recursos necesarios para un adecuado desempeño. En otras palabras, significa que los administradores conociendo la existencia de tal incertidumbre, se verán más motivados a tomar las acciones estratégicas que hagan posible reducirla, absorberla o eliminarla (Kamps y Pólos, 1999; Jabnoun et al., 2003).

Ahora bien, determinar el grado de incertidumbre proveniente del entorno implica su evaluación previa. La misma puede producirse en las mentes de la coalición dominante -gerentes y directivos-, mediante la aplicación de filtros cognitivos durante el proceso de percepción del entorno (Miles y Snow, 1978; Milliken, 1987; Child, 1972, 1997; Walton y Dawson, 2001; Zhang et al., 2012; Ogunsiji y Akanbi, 2013; Zabkar et al., 2013). De este modo, una opción es que la disponibilidad de recursos y capacidades, tanto internas como externas, es producto, en parte, de una acción subjetiva percibida e interpretada por quienes administran y dirigen a la organización. Dichas percepciones, pueden estar influidas por las características intrínsecas o predisposiciones mentales del individuo; es decir, por la construcción previa en sus mentes de sesgos y mapas cognitivos (Zapata y Canet, 2009). La percepción, por tanto, contribuirá a la formación de la visión directiva vinculada con la forma cómo la 
empresa debe ser organizada, estructurada, dirigida y planificada, y cómo las estrategias deben ser formuladas y puestas en marcha para responder adecuadamente, tanto a las circunstancias del entorno como a las necesidades particulares de cada organización. Por ejemplo, la adquisición de recursos, su generación y mantenimiento dentro de ella.

\section{La idea de complementariedad de ambos enfoques teóricos}

Se inicia este apartado con una pregunta: ¿es posible mantener una ventaja competitiva basada únicamente en un conjunto particular de recursos y capacidades dentro de la organización, sin considerar de manera significativa, por un lado los efectos del entorno y la incertidumbre asociado a éste, y, por otro, las limitaciones que, por su propia naturaleza, tienen las organizaciones?

La idea expresada por MartínezMiguélez (2009:38), permite comenzar a esbozar una respuesta: "El principio epistémico de complementariedad subraya la incapacidad humana de agotar la realidad con una sola perspectiva, con un solo punto de vista o con un solo enfoque". Esa idea toca el concepto de racionalidad limitada inherente en el individuo cuando intenta abordar los problemas complejos a los que se enfrenta.

Una de las críticas que se le otorga a la visión basada en recursos, es que se ha centrado fundamentalmente en el análisis de los recursos y capacidades que posee la organización, ignorando de manera significativa el contexto social en el cual se enmarcan las decisiones necesarias para la selección adecuada de tales recursos (Oliver; 1997). Sin embargo, el mismo autor manifiesta que dentro del marco de la TRC el sostenimiento de las ventajas competitivas es el resultado de la elección racional y discrecional de los gerentes, la acumulación y desarrollo de recursos dentro de la organización y los factores estratégicos del sector industrial donde se desenvuelve ${ }^{7}$. Aquí, también se observa que subyace la idea de racionalidad limitada y, además, de alguna manera la presencia, algo tenue, del entorno como un factor condicionante.

Dentro del ámbito de las alianzas estratégicas, Tushar y Teng (2000) hacen referencia al hecho que la TRC no había sido sistemáticamente aplicada en esta área de estudio. Sin embargo, proponen un marco general de la teoría basada en recursos de las alianzas interorganizacionales, notando que las características de algunos recursos tales como: movilidad imperfecta, la no imitación y la poca posibilidad de sustitución incrementan el valor y la motivación para crear alianzas y acuerdos de cooperación.

Reforzando las ideas anteriores, Aragon y Sharma (2003) también reconocen los efectos de los factores externos dentro del marco de los lineamientos de la TRC. Los autores mencionados, en su 
trabajo intentan hacer una integración de la literatura de las teorías: contingente, capacidades dinámicas, y recursos y capacidades; explican cómo ciertas características del entorno -la incertidumbre y la complejidad-, sirven de moderadores en la relación que se da entre las capacidades dinámicas, las estrategias proactivas hacia el entorno y sus consecuencias en la generación de ventajas competitivas. Se deriva de su estudio, un modelo contingente de la visión basada en recursos el cual es verificado empíricamente en Sharma et al., $(2007)^{8}$.

De igual forma, Aragon et al., (2008) encuentran que ciertas capacidades desarrolladas en las empresas, partiendo de sus propios recursos, tales como las estrategias proactivas y la continua innovación, están asociadas con las propiedades del entorno. Por su parte, Song et al., (2005) reconoce que el análisis de los recursos de la organización no puede ser limitado únicamente a sus características internas, tal como lo plantea la teoría basada en recursos, puesto que una buena parte de ellos están fuera de sus fronteras. En todo caso, el autor mantiene un punto de vista de equilibrio o complementariedad entre ambos enfoques.

En su momento, Priem y Butler (2001) plantearon algunas críticas al carácter estático concedido por los investigadores a la visión basada en recursos. En este sentido, muchas configuraciones de recursos pueden generar el mismo valor para diferentes empresas, lo que significa entonces que no necesariamente representan fuentes de ventajas competitivas. Esto es debido a que el valor de los recursos puede variar en la medida que cambia el entorno competitivo; es decir, es en parte una fuente externa la que lo determina.

Ahora bien, Simon (1947) al ubicarse dentro del campo de la psicología cognitiva, donde hay una corriente del pensamiento administrativo que explica los diseños estructurales a través de los procesos cognitivos (Zapata y Canet, 2009), plantea la discusión sobre la racionalidad limitada del individuo que abre caminos importantes en la discusión acerca de la compresión del porqué los individuos actúan y deciden de una manera determinada frente a unas circunstancias dadas, $y$, en consecuencia, en la forma cómo gestionan los recursos de la organización. Así, Simon (1947:24) indica que "la teoría del management, es la teoría de la racionalidad intencionada y limitada del comportamiento de los seres humanos que se dan por satisfechos, puesto que no tienen la inteligencia necesaria para conseguir el máximo (...), el ser humano en su intento por alcanzar la racionalidad se ve restringido dentro de los límites de sus propios conocimientos". En el mismo orden de ideas, Weick (1969), en su trabajo seminal sobre psicología cognitiva y la or-

Las empresas con posición estratégica proactiva, adaptan sus procesos administrativos y de ingeniería para mantener una posición de liderazgo a través del seguimiento continuo de las oportunidades que ofrece el entorno, en lugar de reaccionar repentinamente a los cambios que se presentan en él (Sharma et al., 2007). 
ganización, expresa que la racionalidad limitada se basa en las limitaciones inherentes en el individuo durante el proceso cognitivo de la percepción y procesamiento de la información ${ }^{9}$.

Por otra parte, Schreyogg (1980) y Whittington (1988), en sus trabajos clásicos, planteaban que los postulados básicos que soportan el modelo de adaptación-enfoques contingente y dependencia de recursos-son difíciles de defender en su totalidad, debido al hecho que no consideran prioritario lo que perciben y piensan los gerentes y directivos sobre la manera cómo diseñar y organizar a la empresa. Además, subrayan dichos autores, la perspectiva de adaptación apoya la idea que el entorno es considerado como dado, lo cual se traduce en pocas posibilidades para influir o controlar su situación frente a él. No obstante, dejaron claro que los tomadores de decisiones estratégicas, no son totalmente autónomos ni poseen la capacidad suficiente para enfrentar los problemas solo con los recursos y capacidades de la organización. Esto es porque de alguna manera siempre estará presente la necesaria adaptación a determinados eventos que se suceden en el entorno donde se desenvuelve.

En esa misma línea crítica y también dentro del marco de la teoría neoclá- sica del diseño organizativo, Miles y Snow (1984) señalaban que en ciertas circunstancias, no sólo el entorno ocasiona el deterioro del fit-o desajustes de las variables estructurales ${ }^{10}$-, sino también ocurren desajustes cuando los gerentes y directivos deciden de manera voluntaria hacer cambios en la estrategia, la estructura organizativa y en los procesos internos, debido a un interés particular por participar en nuevos mercados o innovar en tecnología, productos y/o servicios. Se nota aquí una concepción deliberada y explícita de las acciones de quienes toman decisiones estratégicas, ubicándose esto dentro de la perspectiva del "voluntarismo". Perspectiva que ve al individuo (gerentes, directivos y empleados), como la unidad básica de análisis y fuente principal de cambios en la organización, considerándolo como agente autónomo, pro-activo y auto-dirigido (Astley y Van de Ven, 1983; Whittington, 1988; Peris et al., 2006).

Reforzando la ideas anteriores, y dentro de la misma línea de los trabajos clásicos de Astley y Van de Ven (1983), Schreyogg (1980) y Whittington (1988), se encuentran los planteamientos de Child (1972, 1997) y Child et al., (2003) en relación a su perspectiva de la elección estratégica-strategic choice- Este autor señala que para comprender y definir de

9 Williamson (1973: 317) en su trabajo sobre mercado y jerarquía, se refiere a la racionalidad limitada como "los límites de la capacidad del individuo para recibir, almacenar, recuperar y procesar información sin errores".

10 Se refieren a las variables de diseño organizativo: centralización, formalización, especialización del trabajo, ámbito de control, puestos de trabajo, mecanismos de coordinación y sistemas de incentivos. 
una manera más precisa el diseño de la organización y su desempeño, no es suficiente con explicar y establecer relaciones y ajustes adecuados entre las variables internas de la organización y los factores contingentes, sino que también es oportuno considerar las acciones, elecciones y formas de pensamiento de los gerentes y directivos. Esto es, que sus decisiones pueden estar motivadas por su propio interés político, necesidad de poder, sus creencias personales y colectivas, su formación y experiencia, así como también por los procesos de percepción e interpretación del entorno y los procesos cognitivos que tienen lugar en sus mentes. Con lo cual, retendrán cierta discrecionalidad al elegir las acciones que consideren más oportunas y necesarias para alinear los recursos y capacidades de la organización frente a las oportunidades y ventajas que ofrecen los factores contextuales. Cabe destacar que en Venezuela, estos factores se encuentran marcados fuertemente por la incertidumbre reinante

De este modo, las organizaciones responden, en parte, a las percepciones subjetivas de sus gerentes sobre las condiciones del entorno y en especial su incertidumbre, en lugar de considerar únicamente sus características objetivas -determinismo del entorno-. Así, la incertidumbre del entorno se constituye en uno de los principales problemas a enfrentary resolver por la dirección de una organización. En ese sentido, una tendencia importante de la literatura da cuenta que mientras mayor es la percepción de incertidumbre, los gerentes y directivos prestarán mayor atención a sus políticas y estrategias, y en general, a todos aquellos procesos y acciones que permitan mejorar las capacidades organizacionales (Miles y Snow, 1978; Milliken, 1987; Agbejule, 2005; Rhys, 2008; Kanarattanavong y Ruenrom, 2009; López et al., 2011). Es un tema central en la teoría de la organización el estudio de la percepción de la incertidumbre del entorno-perceived environmental uncertainty-, constituyéndose en una variable intermedia que enlaza el entorno con los procesos organizacionales, la estructura organizativa y el desempeño organizacional.

Sobre la base de las consideraciones anteriores, se comparte la idea que bajo ciertas condiciones contextuales parecidas y ubicadas en el mismo sector industrial o comercial, las organizaciones pueden elegir políticas, acciones y estrategias muy diferentes para alcanzar sus objetivos. Incluso dentro de la misma organización, los gerentes tienen percepciones y visiones distintas sobre como estructurarla y dirigirla. Así, se destaca la idea ecléctica de que el manejo adecuado de los recursos y capacidades y su integración con aquellos ofrecidos por el entorno y de las ventajas competitivas que se derivan de ello, constituyen una cuestión de vital importancia para la organización. Lo que significa que su supervivencia no solo está condicionada por las características objetivas internas -tecnología, equipos, espacios físicos, recursos humanos-y por la naturaleza del entorno -factores de contingencia-, sino también por la visión e interpretación de sus gerentes y directivos acerca de los acontecimientos que suceden dentro y alrededor de ella. Esto es lo que denominamos visión directiva, donde se establece como premisa fundamental que los dirigentes 
de la organización pueden elegir las decisiones más convenientes y oportunas por encima incluso de las circunstancias del entorno, y de otros factores internos de acuerdo a sus preferencias perceptivas, no obstante, reconoce su presencia objetiva. Sin embargo, su visión subjetiva se logra a través del proceso cognitivo de la percepción (Peris, et al., 2006; Zapata et al., 2008).

La perspectiva de la visión directiva, plantea que no sólo los factores del entorno y los recursos restringen y determinan el diseño y comportamiento de la organización. También hay otros factores intrínsecos en el individuo, como los estilos de liderazgo, los mapas y sesgos cognitivos y las percepciones que influyen en las características y formas de gestión empresarial (Child, 1997; Hodgkinson et al., 2004; Keil et al., 2007; Thomas et al., 2007; Zapata et al., 2008, Zapata y Canet, 2009). Formas de gestión que se traducen: a) en el establecimiento de las metas, los objetivos, la misión, la visión, las estrategias, los procesos de conversión y la estructura organizativa, y b) en la definición de los productos, los mercados o sectores donde participará, y la tecnología, los conocimientos y los recursos -físicos, financieros y humanos- que empleará.

Bajo los planteamientos anteriores, se sostiene entonces que el desarrollo de las capacidades para generar recursos diferenciados $\mathrm{y}$, como consecuencia de ello, ventajas competitivas, forma parte de un proceso de percepción e interpretación individual y colectiva de la construcción y reconstrucción del contexto interno y externo de la organización en las mentes de los individuos que la administran y la dirigen.

\section{Modelo teórico y proposiciones planteadas}

El modelo propuesto objeto de este trabajo, se construye sobre las bases de dos enfoques teóricos divergentes pero que se consideran a la vez complementarios. Por lo tanto, se intenta integrarlos con el propósito de mitigar las limitaciones de sus propios puntos de vista y postulados cuando explican el origen de los recursos y capacidades de la organización y como consecuencia de ello las ventajas competitivas.

Se busca entonces, un sentido de equilibrio entre el enfoque teórico -recursos y capacidades- que centra el éxito de la organización en sus características internas y aquel que la considera fundamentalmente como un sistema adaptativo y reactivo a las circunstancias objetivas del entorno donde se desenvuelve -dependencia de recursos-. En todo caso, de lo que se trata es de hacerlos más útiles cuando se intenta comprender el proceso de acumulación y generación de recursos y capacidades y su relación con los conceptos de racionalidad limitada y visión directiva presentes en dinámica diaria de la organización.

En Diagrama 1 se presenta el modelo teórico propuesto que intenta dar un sentido de integración y complementariedad a los planteamientos de los enfoques teóricos abordados.

Las organizaciones requieren de recursos y capacidades que produzcan ventajas competitivas que le permitan satisfacer, tanto sus demandas y necesidades internas como las externas provenientes del entorno, y de este modo de poder crecer, adquirir ventajas competitivas o de simplemente sobrevivir. Para al- 


\section{Diagrama 1 \\ Modelo Teórico Integrado del Origen de los Recursos y Capacidades de la Organización}

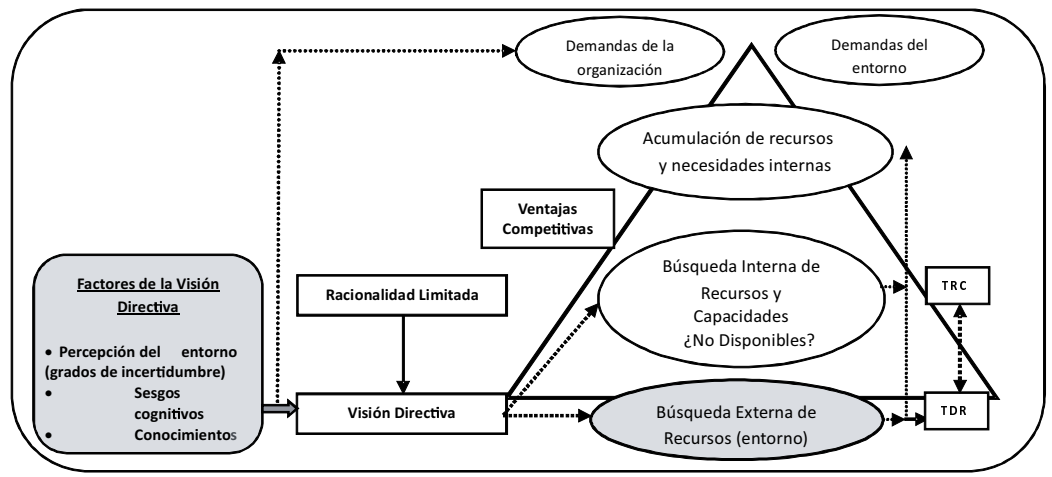

Fuente: Elaboración propia.

canzar esos objetivos, es determinante el hecho de tomar decisiones oportunas acerca de cómo utilizar los recursos y capacidades con los que cuenta internamente, o de cómo gestionarlos y encontrarlos fuera de la organización. De este modo, la organización en primera instancia examina su existencia dentro sí misma, estando su disponibilidad condicionada: (a) por la acumulación histórica y la maduración de sus procesos formalizados a través de rutinas organizativas; (b) por su capacidad para producirlos o de implementar estrategias que permitan su acumulación y mantenimiento, estando ambos procesos restringidos por la racionalidad limitada, tanto individual como colectivo; y (c) por la visión de los gerentes y directivos en cuanto a la manera de gestionar dichos recursos y capacidades. La misma, puede estar condicionada por factores tales como: sesgos cognitivos, el conocimiento, los valores y la percepción del entorno, en especial por su nivel de incertidumbre.
Ahora bien, si esas condiciones no permiten obtener los recursos necesarios y la organización no tiene la suficiente capacidad de integración vertical para lograrlos, entonces se verá en la necesidad de recurrir a la disponibilidad de recursos que existen en su entorno. Ello a través de la conexión de redes externas que le provean el flujo de recursos y capacidades necesarios y le sirvan de complemento a los que internamente posee. En ese sentido, Gulati et al., (2000) subrayan que el potencial de las redes estratégicas de cooperación se debe al hecho de que por medio de ellas las empresas tienen acceso a una variedad de información, recursos, mercados y tecnologías. El resultado es un conjunto de recursos y capacidades de difícil imitación por los competidores. Así, una vez que el recurso ingresa a la organización sufre un proceso de transformación, que de acuerdo a sus propias capacidades y limitaciones, así como también por el efecto de la visión directiva, puede convertirse en poco imita- 
ble, escaso y, por ende, esencial. Esto puede traer consigo mayores ventajas competitivas y características diferenciadoras con respecto a otras organizaciones.

La situación planteada ocasiona costos adicionales debido a: (a) un mayor grado de dependencia de factores del entorno no controlados por la organización; y (b) la incorporación de estrategias y acciones que contribuyan a controlarlos o al menos disminuir esa dependencia.

Finalmente, del modelo planteado en la Figura 1 y de las reflexiones realizadas sobre los enfoques teóricos objetos de estudio de esta investigación, se desprenden las siguientes cinco proposiciones que posteriormente podrán ser contrastadas en una investigación de carácter empírico.

(P1):"Las capacidades de la organización, están condicionadas tanto por la racionalidad limitada como por la visión directiva de los gerentes y directivos".

(P2):"La visión directiva y la racionalidad limitada de los gerentes y directivos, condicionan la relación entre los recursos y capacidades y las ventajas competitivas de la organización".

(P3):"La búsqueda de recursos y su disponibilidad dentro de la organización, está condicionada tanto por la racionalidad limitada como por la visión directiva de los gerentes y directivos".

(P4):"La búsqueda de recursos y su disponibilidad en el entorno de la organización, está condicionada por la visión directiva y la racionalidad limitada los gerentes y directivos".
(P5):"Entre organizaciones de un mismo sector y bajo condiciones de entorno muy semejantes, la visión directiva y la racionalidad limitada generan diferencias significativas en su capacidad para producir ventajas competitivas".

En términos generales, estas proposiciones quedan enmarcadas dentro de los postulados de la teoría contingente (determinismo-contingente), puesto que no solo cubren aspectos subjetivos, inherentes e intrínsecos al individuo, que influyen en las decisiones directivas y gerenciales relacionadas con la búsqueda de recursos y capacidades necesarios para que la organización funcione y cumpla con sus objetivos, sino que también se consideran dos aspectos. Primero, los factores contingentes objetivos externos que definen al entorno como un conjunto de recursos y procesos que restringen, limitan y controlan a la organización (tales como: la innovación y la tecnología, las demandas de los clientes, condiciones del mercado, las exigencias gubernamentales, los procesos políticos, el marco institucional y la localización de la organización). Segundo, los factores contingentes internos objetivos, tales como: el tamaño y la edad de la organización, sus procesos administrativos y tecnológicos y plan estratégico. Factores todos que deben alcanzar un nivel adecuado de ajuste con las variables de diseño organizativo a través de las decisiones directivas y gerenciales a fin de poder lograr ventajas competitivas diferenciadoras $y$ desempeños superiores. 


\section{Conclusiones}

En el desarrollo de este artículo se ha hecho un esfuerzo por ordenar, armonizar e integrar las distintas propuestas teóricas más notables que explican desde sus puntos de el origen de los recursos y capacidades de la organización, en este caso referido principalmente a las teorías de recursos y capacidades y de dependencia de recursos.

Como consecuencia de ello, se logró plantear un modelo teórico que intenta integrar con un sentido de complementariedad los postulados que orientan a ambas teorías explicativas del origen de los recursos y capacidades de la organización y, el establecimiento de un grupo de proposiciones que podrán ser contrastadas posteriormente en investigaciones de carácter empírico. Dichas proposiciones señalan que los procesos de toma de decisiones relacionados con la elección y adquisición de los recursos que requiere la organización para alcanzar ventajas competitivas y competencias distintivas con respecto a otras organizaciones dependen de los procesos racionales orientados por principios administrativos y limitados por la capacidad de aprendizaje y análisis del individuo, y la visión del directivo o del gerente. Esta última expresada a través de su acción voluntaria, deliberada y discrecional en cuanto a la manera como debe ser diseñada, organizada y dirigida la empresa. Cuestión esta que puede estar condicionada por factores tales como: los sesgos cognitivos y las predisposiciones mentales, la capacidad de percepción, los valores, el conocimiento, los estilos de liderazgos y la cultura. En general, son factores que contribuyen a la forma como los gerentes y directivos comprende el mundo donde se desenvuelven y en la manera como toman las decisiones en la empresas en el orden estratégico, administrativo y operativo.

Se observa que las teorías analizadas, tienen un carácter heterogéneo entre ellas en función a la manera como explican y abordan, por medio de sus postulados y principios, los orígenes de los recursos y capacidades de las organizaciones; pero a su vez, proporcionan en sí mismas un valor relevante en la amplitud de los diferentes aspectos que abordan y que tratan de explicar. Por tanto, es más importante y útil emplearlas como fundamentos teóricos complementarios y no excluyentes como tradicionalmente han sido tratadas y aplicadas cuando se evalúa el origen y desarrollo de los recursos y capacidades de la organización, o cuando se intenta encontrar respuestas adecuadas a los problemas de la organización, tanto en el orden estratégico como operativo. Contribuyendo, asimismo, a explicar su comportamiento, su estructura y sus procesos, organizativos. Por tanto, se convierte así está discusión en una contribución académica al proceso de comprensión del desarrollo de las organizaciones y al planteamiento de soluciones a sus problemas frente a determinadas circunstancias externas e internas.

Ambos enfoques teóricos, tienen efectos estratégicos y operativos que proveen diferentes formas de comprender el comportamiento y los resultados de la organización, no son incompatibles entre ellas sino complementarias en la explicación del origen de los recursos y capacidades de la organización y, como consecuencia, las ventajas competitivas sostenibles en el tiempo. 


\section{Referencias bibliográficas}

Abenson, Felix y Taku, Michael (2006). Knowledge Source and Small Business Competitiveness. Competition Forum, Vol. 4, No. 2, pp. 464-469.

Agbejule, Adebayo (2005). The Relationship Between Management Accounting Systems and Perceived Environmental Uncertainty on Managerial. Accounting and Business Research, Vol. 23, No. 4, pp. 295-305.

Aldrich, Howard y Pfeffer, Jeffrey (1976). Environments of Organizations. Annual Reviews of Sociology, Vol. 2, pp. 79-105.

Akingbola, Kunle (2013). Contingency, fit and flexibility of HRM in Nonprofit Organizations. Employee Relations, Vol. 25, No. 5, pp. 479-49.

Astley, Graham y Van de Ven, Andrew (1983). Central Perspective and Debates in Organizations Theory. Administrative Science Quarterly, Vol. 28, No. 2, pp. 245-273.

Aragón-Correa, Alberto y Sharma, Sanjay (2003). A contingent Resource-Based View of Proactive Corporate Environmental Strategy. Academy of Management Review, Vol. 28, No. 1, pp. 71-88.

Aragon-Correa, Alberto; Hurtado-Torres, Nuria.; Sharma, Sanjay y García-Morales, Victor (2008). Enviromental Strategy and Performance in Small Firms: A Resource-Based Perspective. Journal of Enviromental Management, Vol. 86, pp. 88-103.

Barney, Jay (1986). Strategic Factor Markets: Expectations, Luck and Business Strategy. Management Science, Vol. 31, pp. 1231-1241.

Barney, Jay (1991). Firm Resources and Sustained Competitive Advantage. Journal of Management, Vol. 17, No. 1, pp. 99-120.
Barney, Jay; Wright, Mike y Ketchen, David (2001). The Resource-based View of the Firm: Ten Years After 1991. Journal of Management, Vol. 27, pp. 625-641.

Barney, Jay (2001). Is the Resource Based "View" a Useful Perspective for Strategic Management Research? Yes. Academy of Management Review, Vol. 26, No. 1, pp. 41-56.

Barney, Jay y Arikan, Asli Musaoglu (2001). The Resource-Based View: Origins and Implications. En M.A Hitt R.E Freeman \& J.S Harrison (Ed.), The Blackwell Handbook of Strategic Management.

Bingham, Christopher y Eisenhardt, Kathleen (2008). Position, Leverage and Opportunity: A Typology of Strategic Logics Linking Resources with Competitive Advantage. Managerial and Decision Economics, Vol. 29, pp. 241-256.

Blome, Constantin; Schoenherr, Tobias y Eckstein, Dominik (2014). The impact of knowledge transfer and complexity on supply chain flexibility: A knowledge-based view. International Journal of Production Economics. Vol. 147, No. Part B, pp. 307-316.

Bryant, Phil (2011). Regulated Change Effects on Boards of Directors: A Look at Agency Theory and Resource Dependency Theory. Proceedings of the Academy of Strategic Management, Vol. 10, No. 1, pp.19-23.

Child, John (1972). Organizational Structure, Environment and Performance: The Role of Strategic Choice. Sociology, Vol. 6, pp. 1-22.

Child, John (1997). In the Analysis of Action, Structure, Organization and Environment: Retrospect and prospect strategic choice in the analysis of action, structure, Organization and Environment. Retrospect and Prospect. Organization Studies, Vol. 8, No. 1, pp. 43-76. 
Child, John; Lleanne, Chung y Howard, Davies (2003). The Performance of CrossBorder Units in China: A Test of Natural Selection, Strategic Choice and Contingency Theories. Journal of International Business Studies, Vol. 43, No. 3, pp. 242-254.

Conner, Kathleen y Prahalad, C.K. (1996). A Resource-based Theory of The Firm: Knowledge vs. Opportunism. Organization Science, Vol. 7, No. 5, pp. 477-501.

Coleman, Susan; Cotei, Carmen y Farhat, Joseph (2013). A Resource-Based View of New Firm Survival: New Perspectives on The Role of Industry and Exit Route. Journal of Developmental Entrepreneurship, Vol. 18, No. 1-25

Collis, David (1994). How Valuable are Organizational Capabilities?. Strategic Management Journa, Vol. 15, pp. 143152.

Day, George (1994). The Capabilities of Market-Driven Organizations. Journal of Marketing, Vol. 58, pp. 37-52.

Dierickx, Ingemar y Cool, Karel (1989). Asset Stock Accumulation and Sustainability of Competitive Advantage. Management Science, Vol. 35, No. 2, pp. 1504-1513.

Donaldson, Lex (2001). The Contingency Theory of Organizations. London: Sage Publications.

Duh, Mojca (2010). Applying Agency Theory and the Resource-Based View in Explaining Performance Differences Between Family and Non-family Businesses. Our Economy (Nase Gospodarstvo), Vol. 56, No. 1/2, pp. 45-52.

Escandón-Barbosa, Diana y Hurtado-Ayala, Andrea (2013). Internacionalización de Empresas bajo la Perspectiva de Recursos y Capacidades: Caso Sector Autopartes. Cuadernos Latinoamericanos de Administración, Vol. 9, No. 17, pp. 72-85.
Esteve-Pérez, Silviano y Mañez-Castillejo, Juan (2008). The Resource-Based Theory of the Firm and Firm Survival. Small Business Economics, Vol. 20, pp. 231-249.

Fink, Robert.; Edelman, Linda; Hatten, Kenneth y James, Willian (2006). Transaction Cost Economics, Resource Dependence Theory, and Customersupplier Relationships. Industrial and Corporate Change, Vol.15, No. 3, pp. 497-529.

Gaur, Ajai; Kumar; Vikas y Singh, Deeksha (2014). Institutions, Resources, and Internationalization of Emerging Economy Firms. Journal of World Business, Vol. 49, pp. 12-20.

Gopalakrishnan, Shanthi y Dugal, Mohinder (1998). Strategic Choice versus Environmental Determinism: A Debate Revisited. International Journal of Organizational Analysis, Vol. 6, No. 2, pp. 146-164.

Gulati, R; Nohria, N. y Zaheer, A. (2000). Strategic Networks. Strategic Management Journal, Vol. 21, pp. 203-215.

Gupta, Vishal; Huang, Rui; Yayla, Ali (2011) Social Capital, Collective Transformational Leadership, and Performance: A Resource-Based View of SelfManaged Teams. Journal of Managerial Issues, Vol. 23, No. 1, pp. 3145.

Grant, Robert (1991). The Resourses-Based Theory of Competitive Advantage: Implications for Strategy Formulation. California Management Review, Vol. 22, pp. 114-135.

Grant, Robert (1996). Prospering in Dynamically-Competitive Environments: Organizational Capability as Knowledge Integration. Organization Science, Vol. 7, No. 4, pp. 375-387.

Halawi, Leila; Aronson, Jay y McCarthy, Richard (2005). Resource-Based View of Knowledge Management for Com- 
petitive Advantage. Electronic Journal of Knowledge Management, Vol. 3, No. 2, pp. 75-80.

Hamel, Gary y Prahalad, C.K. (1995). Compitiendo con el Futuro. Barcelona: Editorial Ariel

Han, Yongliang (2007). Populations Ecology, Strategic Choice, and Competition. International Journal of Business Research, Vol. VII, No. 4, pp. 124128.

Hannan, Michael y Freeman, John (1977). The Population Ecology of Organizations. American Journal of Sociology, Vol. 82, pp. 929-964.

Hannan, MichaeL y Freeman, John (1987). The Ecology of Organizational Founding: American Labor 1836-1985. American Journal of Sociology, Vol. 92, pp. 910-943.

Helfat, Constance y Peteraf, Margaret (2003). The Dynamic Resource-Based View: Capability Lifecicles. Strategic Management Journal, Vol. 24, pp. 9971010.

Hou, Jia-Jeng (2008). Toward a Research Model of Market Orientation and Dynamic Capabilities. Social Behavior and Personality, Vol. 36, No. 9, pp. 1251-1268.

Hodgkinson, Gerard; Maule, John y Bown, Nicola (2004). Causal Cognitive Mapping in the Organizational Strategy Field: A Comparison of Alternative Elicitation Procedures. Organizational Research Methods, Vol. 7, No. 1, pp. 3-26.

Hoopes David; Madsen, Tammy, Walker, Gordon (2003). Why in there a resourced-based view?. Toward a Theory of Competitive Heterogeneity. Strategic Management Journal, Vol. 24, pp. 889-902.

Hsu, David y Zledonis, Rosemarie (2013). Resources as Dual sources of Advantage: Implications for Valuing Entrepre- neurial-firm Patents. Strategic Management Journal, Vol. 34, pp. 761781.

Jabnoun, Naceur; Khalifah, Azaddinn y Yusuf, Attahr (2003). Environmental Uncertainty, Strategic Orientation, and Quality Management: Contingency Model. The Quality Management Journal, Vol. 14, No. 4, pp. 17-30.

Kamps, Jaap y Pólos, László (1999). Reducing Uncertainty: A Formal Theory of Organizations in Action. American Journal of Sociology, Vol. 104, No. 6, pp. 1776-1812.

Kanarattanavong, Apichart. y Ruenrom, Guntalee (2009). The Model of Corporate Environmentalism: the Effects of Perceived Market Uncertainty upon Marketing, Environmental, and Social Performance. The Business Review, Cambridge, Vol. 12, No. 2, pp. 140147.

Keil, Mark; Gordon, Depledge y Rai, Arun (2007). Escalation: The Role of Problem Recognition and Cognitive Bias. Decision Sciences, Vol. 38, No. 3, pp. 391-421.

Knott, Paul (2009). Integrating Resource Based Theory in a Practice Relevant Form. Journal of Strategy and Management, Vol. 2, No. 3, pp. 163-174.

Langlois, Richard y Foss, Nicolai (1999). Capabilities and Governance: The Rebirth of Production in the Theory of Economic Organization. Kyklos, Vol. 52, No, 22, pp. 201-218.

Lei, David (2003). Competition, Cooperation and Learning: The New Dynamics of Strategy Organisation Design for the Innovation Net. International Journal Technology Management, Vol. 26, pp. 694-712.

López-Gamero, María; José, Molina-Azorín; Claver-Cortés, Enrique (2011). Environmental uncertainty and environmental management perception: A 
multiple. Case Study..Journal of Business Research, Vol. 64, pp. 427435.

Martínez-Miguélez, Miguel (2009). Nuevos Paradigmas en la Investigación. Caracas: Alfa.

Makadok, Richard (2001). Toward a Synthesis of The Resource-Based and Dynamic-Capability Views of Rent Creation. Strategic Management Journal, Vol. 22, No. 5, pp. 387-401.

Miles, Raymond y Snow, Charles (1978). Organizational Strategy, Structure, and Process. McGraw-Hill Publishing Company.

Miles, Raymond y Snow, Charles (1984). Fit, Failure and The Hall of Fame. California Management Review, Vol. 26, No. 3, pp. 10-28.

Milliken, Frances (1987). Three types of Uncertainty About the Environment: State, Effect and Response Uncertainty. Academy of Management Review, Vol. 12, No. 1, pp.133-143.

Molloy, Janice; Chadwick, Clint, Lloyhart, Robert y Golden, Simon (2011). Making Intangibles 'Tangible"' in Tests of Resource-Based Theory: A Multidisciplinary Construct Validation Approach. Journal of Management. Vol. 37, pp. 1496-1518

Morris, Michael; Kuratko, Donald; Allen, Jeffrey, Ireland y Duane; Schindehutte (2010). Resource Acceleration: Extending Resource-Based Theory in Entrepreneurial Ventures. Journal of Applied Management and Entrepreneurship, Vol. 15, No. 2, pp. 4-25.

Nienhüser, Werner (2008). Resource Dependence Theory-How Well Does It Explain Behavior of Organizations? Management Revue, Vol. 19, No. 1/2, pp. 9-32.

Nonaka, Ikujiro (1994). A Dynamic Theory of Organizational Knowledge Creation.
Organization Science, Vol. 5, No. 1, pp. 14-27.

Nonaka, Ikujiro y Takeuchl, Hirotaka (1995). The Knowledge Creating Company. How Japanese Companies Create the Dynamic of Innovation. New York: Oxford University Press

Nonaka, Ikujiro y Toyama, Ryoko (2005). The Theory of the Knowledge-Creating Firm: Subjectivity, Objectivity and Synthesis. Industrial and Corporate Change, Vol. 14, No. 3, pp. 419-436.

Ogunsiji, Amos Sola y Akanbi, Paul Ayobami (2013). The Role of Perceived Environmental Uncertainty and Strategic Agility on the Performance of Selected Banks in Oyo State of Nigeria. Information and Knowledge Management, Vol.3, No.7, pp. 23-30.

Oliver, Christine (1997). Sustainable Competitive Advantage: Combining Institutional and Resource Based View. Strategic Management Journal, Vol. 18, No. 9, pp. 697-713.

Penrose, Edith (1959). The Theory of the Growth of the Firm. Wiley: New York

Peterson, Douglas (2011). Partner Selection for Corporate Social Responsibility Efforts: The Case of Choosing NGO Partners Using Transaction Cost Analysis and Resource Dependency. Interdisciplinary Journal of Contemporary Research in Business, Vol. 2, No. 11, pp. 12-22.

Peteraf, Margaret (1993). The Cornerstones of Competitive Advantage: A Resource Based View. Strategic Management Journal, Vol. 14, No. 3, pp. 179-191.

Peris, Fernando.; Peris-Ortiz, Marta y ZapataRotundo, Gerardo, J. (2006). Complementariedad de Teorías en la Administración Eficiente del Trabajo: Un Análisis Microorganizativo, Compendium, Vol. 17, pp. 75-94.

Peng, George y Beamish, PauL (2014). MNC Subsidiary Size and Expatriate Con- 
trol: Resource-Dependence and Learning Perspectives. Journal of World Business, Vol. 49, No. 1, pp. 51-62.

Pfeffer, Jeffrey y Salancik, Gerald (1978). The External Control of Organizations: A Resource Dependence Perspective. Harper \& Row, Publishers.

Porter, Michael (1982). Estrategia Competitiva, México: CECSA.

Prahalad, Coimbatore Krishnarao y Hamel, Gary (1990). The Core Competence of the Corporation. Harvard Business Review, Vol. 68, pp. 79-91.

Priem, Richard y Butler, John (2001). Is the Resource Based View a Useful Perspective for Strategic Management Research. Academy of Management Review, 26 (1), 22-40.

Rao, Madhu; Brown, Carol y Perkins, William (2007) Host Country Resource Availability and Information System Control Mechanisms in Multinational Corporations: An Empirical Test of Resource Dependence Theory. Journal of Management Information Systems, Vol. 23, No. 4, pp. 11-28.

Ray, Gautam; Muhanna, Waleed y Barney, Jay (2005). Information technology and the performance of the customer service process: A resource-based analysis. MIS Quarterly, Vol. 20, No. 4.

Ray, Gautam; Barney, Jay y Muhanna, Waleed (2004). Capabilities Processes and Competitive Advantage: Choosing the Dependent Variable in Empirical Test of The Resource-Based View. Strategic Management Journal, Vol. 25, pp.23-37.

Rhys Andrews (2008). Perceived Environmental Uncertainty in Public Organizations An Empirical Exploration. Public Performance \& Management Review, Vol. 32, No. 1.

Selznick, Philip (1957). Leardership in Administration: A Sociological Perspective, en Foss, N. J. (ed., 1997): Resources, Firms and Strategies. A Rea- der in the Resource-Based Perspective. Oxford University Press, pp. 2126.

Sharma, Sanjay; Aragon-Correa, Alberto y Rueda-Manzanares Antonio (2007). The Contingent Influence of Organizational Capabilities on Proactive Environmental Strategy in the Service Sector: An Analysis of North American and European Ski Resorts. Canadian Journal of Administrative, Vol. 28, pp. 268-283.

Schreyogg, Georg (1980). Contingency and Choice in Organization Theory. Organizations Studies, Vol. 1, No. 4, pp. 305-326.

Simon, Herbert (1947). Administrative Behavior. Las citas y referencias corresponden a la versión en castellano: EI Comportamiento Administrativo, 1979. Biblioteca de Economía de la Empresa-Aguilar.

Song, Michael Song; Droge, Cornelia; Hanvanich, Sangphet y Calantone, Roger (2005). Marketing and Technology Resource Complementarity: An Analysis of their Interaction Effect in Two Environmental Contexts. Strategic Management Journal, Vol. 26, pp. 259-276.

Thomas, Douglas; Eden, Lorraine, Hitt, Michael y Stewart, Liller (2007). Experience of Emerging Market Firms: The Role of Cognitive Bias in Developed Market Entry and Survival. Management International Review, Vol. 47, No. 6, pp. 845-867.

Tushar Kanti Das y Teng, Bing-Sheng (2000). A Resource-based Theory of Strategic Alliances. Journal of Management, Vol. 20, pp. 31-61.

Ulrich, David y Barney, Jay (1984). Perspectives in Organizations: Resource Dependence, Efficiency, and Population. Academy of Management Review. Vol. 9, No. 3, pp. 471-481. 
Walton, Eric y Dawson, Sarah (2001). Managers' Perceptions of Criteria of Organizational Effectiveness. Journal of Management Studies, Vol. 38, No. 2, pp. 173-199.

Wernerfelt, Biger (1984). A Resource-Based View of the Firm. Strategic Management Journal, Vol. 5, No. 2, pp. 171-180.

Weick, Karl (1969). The Social Psychology of Organizing. Addison-Wesley Publishing.

Wei, Wu. (2006). The Relationship Among Corporate Political Resources, Political Strategies and Political Benefits of Firms in China Based on Resource Dependency Theory. Singapore Management Review, Vol. 2, No. 2, pp. 85-14.

Whittington, Richard (1988). Environment, Structure y Theories of Strategic Choices. Journal of Management Studies, Vol. 25, No. 6, pp. 125-142.

Williamson, Oliver (1973).Organizational Forms and Internal Efficiency. Markets and Hierarchies: Some Elementary Considerations. American Economic Association, Vol. 63, No. 2, pp. 316-325.

Volberda, Henk; Niels, Van Der, Weerdt.; Verwaal, Ernst; Stienstra, Marten y Verdu, Antonio (2012). Contingency Fit, Institutional Fit, and Firm Performance: A Meta fit Approach to Organization-Environment Relationships. Organization Science, Vol. 23, No. 4, pp.1040-1054.

Yang, Yi. (2008). The Roles of Human Resources, Information Technology, and Marketing Knowledge Capabilities in Performance: an Extension of the Resource-Based Theory Perspective. Social Behavior and
Personality, Vol. 26, No. 9, pp. 12691282.

Zabkar, Vesna; Cater, Tomaz; Bajde, Domen; Cater, Barbara (2013). Environmental Strategy: a Typology of Companies Based on Managerial Perceptions of Customer's Environmental Activeness and Deterrents. E+M Ekonomie a Management, Vol.16, No. 3, pp. 57-74.

Zapata Rotundo, Gerardo; Canet, María y Peris-Bonet, Fernando. (2008). Visión Directiva y Elección Estratégica: Su Influencia en los Tipos Básicos de Trabajo. Cuadernos de Administración, Vol. 21, No. 37, pp. 185-209.

Zapata Rotundo, Gerardo y Canet, María (2009). La Cognición del Individuo: Reflexiones sobre sus Procesos e influencia en la Organización. Revista Espacio Abierto: Cuaderno Venezolano de Sociología, Vol. 18, No. 2, pp. 46-62.

Zapata Rotundo, Gerardo y Mirabal, Alberto (2011). El Cambio en la Organización: Un Estudio Teórico desde La Perspectiva de Control Externo. Revista Estudios Gerenciales, Vol. 27, No. 119, pp. 79-98.

Zhang, Xue; Majid, Shaheen y Foo, Schubert (2012). Perceived Environmental Uncertainty, Information Literacy and Environmental Scanning: Towards a Refined Framework. IR Informatiom Research, Vol. 17, No. 2.

Zhuang, Youlong y Lederer, Albert (2006). A Resource-Based View of Electronic Commerce. Information \& Management, Vol. 3, No. 2, pp. 251-261.

Ziesemer, Thomas (2013). A Knowledge-Based View of the Porter Hypothesis. Environmental Policy \& Governance, Vol. 23, No.3, pp. 193-208. 